Clásicos confucianistas, shuyuan, ritos confucianistas, fundamentalista, Jiang Qing

\title{
EL RECIENTE RESURGIMIENTO DEL CONFUCIANISMO POPULAR EN CHINA CONTINENTAL: EL REDESCUBRIMIENTO DE LOS CONFUCIANISTAS CLÁSICOS, LAS ACADEMIAS Y LOS RITOS
}

YONG CHEN

El Colegio de México

Hasta ahora, las tendencias culturales de China durante las últimas tres décadas han presentado temáticas altamente cambiantes. Si la década de 1980 puede llamarse la década de la "fiebre cultural” (wenbua re 文化热), y la de 1990 la década del “aprendizaje de Estado" (gио xиe 国学), ${ }^{1}$ no estaría muy fuera de lugar denominar los primeros diez años del siglo xxi como la década del “resurgimiento confucianista" (ruxue fuxing 儒学复兴). Sin embargo, es necesario tener presente que este renacimiento confucianista, que ha sido bastante difundido, es diferente a la disciplina académica denominada estudios confucianistas (ruxue 儒学) que, como parte integrante de la fiebre "del aprendizaje de Estado", ha formado parte del paisaje intelectual de China desde principios de 1990. A manera de revancha frente a la famosa declaración de Joseph R. Levenson ${ }^{2}$ de que hacia finales de la dinastía Qing (1644-1911) el confucianismo había perdido su iniciativa, y del lamento de Yu Yingshi ${ }^{3}$ de que se ha

Este artículo fue recibido por la dirección de la revista el 30 de abril de 2013 y aceptado para su publicación el 29 de julio de 2013.

${ }^{1}$ Guoxue también se traduce como aprendizaje nacional. Véase Arif Dirlik, "Guoxue/National Learning in the Age of Global Modernity", China Perspectives, núm. 2011/1.

${ }^{2}$ Joseph R. Levenson, Confucian China and its Modern Fate: The Problem of Intellectual Continuity, Berkeley-Los Ángeles, University of California Press, 1968, pp. IX, X.

${ }^{3}$ Yu Yingshi, Xiandai Ruxue Lun [Sobre el aprendizaje confucianista moderno], Shanghái, Shanghai renmin chubanshe, 1998, p. 5. 
convertido en un "fantasma errante", la tranquila pero constante revitalización del confucianismo, como tradición viva, se inicia popularmente y alcanza la conciencia nacional debido a que en la China del siglo XXI surge como un fenómeno cultural fascinante.

El renacimiento confucianista es atestiguado por varios movimientos relacionados entre sí: la campaña para "leer a los clásicos” (dujing 读 经), ${ }^{4}$ el renacimiento de las academias confucianistas (shuyuan 书院), y el resurgimiento de los ritos tradicionales (confucianistas) (chuantong liyi 传统礼仪), entre otros ejemplos. A partir de mediados de la década de 1990 y hasta la fecha, la campaña para "leer a los clásicos" ha involucrado a millones de niños en edad escolar en todo el país; además, se dio en convergencia con un movimiento similar en Taiwan iniciado por Wang Caigui hace aproximadamente dos décadas. Aunque la metodología y los valores que promueve estimularon una gran controversia, la campaña en sí revela el entusiasmo popular para reconectarse con un patrimonio cultural que data de hace miles de años.

El renacimiento de las academias confucianistas (shuyuan 书院) en los últimos años desafía el tedioso debate sobre si el confucianismo es o no es una religión, mientras arraiga en la conciencia pública de manera discreta pero constante. Este renacimiento modela con diligencia a las academias neoconfucianistas de Song-Ming ya que, esencialmente desencantadas con el patrocinio oficial, estas academias de reciente creación están fuertemente comprometidas con una ambición religiosa: diacrónicamente, para continuar con el daotong (道统, el hilo del Tao) de la mano de los neoconfucianistas de Song-Ming; y sincrónicamente, para protegerse de las "herejías", con el cristianismo como el candidato más accesible para tal protección. A diferencia de las academias confucianistas tradicionales, que a menudo estuvieron atrapadas entre lo "privado" y lo "oficial", las academias confucianistas de reciente creación, desde sus inicios han estado muy conscientes de su papel extraoficial y extragubernamental.

${ }^{4}$ Aunque los clásicos a los que nos referimos incluyen algunos fragmentos del taoísmo, del budismo y de otras tradiciones, se trata predominantemente de clásicos confucianistas. 
El más reciente resurgimiento de los ritos tradicionales (confucianistas) sólo ha alcanzado de manera esporádica las noticias nacionales y ha captado menos la atención del público; sin embargo, es el testimonio de un fuerte compromiso con la restauración del confucianismo como tradición holística. Aunque tradicionalmente el confucianismo reclama la posesión de cientos de ritos que establecen las normas con las que se rige la sociedad, hoy en día sólo hemos sido testigos de la restauración de algunos de ellos, como el culto a Confucio, la ceremonia matrimonial confucianista, y la ceremonia del "estreno de los pinceles" (kaibi li 开笔礼, la iniciación a la edad escolar). En contraste con el patrocinio estatal del sacrificio al Emperador Amarillo o a otros ancestros nacionales, lo que sin duda fomenta sentimientos nacionalistas, estos recién restaurados ritos tratan de cumplir firmemente con las normas confucianistas y de adherir seguidores leales.

$\mathrm{Al}$ analizar las manifestaciones del confucianismo en el ámbito popular, como una tradición viva, mi trabajo tiene por objeto investigar los mecanismos sociales que dan origen a este renacimiento confucianista y lo que significa para una sociedad que se moderniza rápidamente y donde ninguna ideología puede tener el control de la conciencia pública. Se argumenta que, con el fin de recuperar su relevancia contemporánea, el confucianismo tiene que participar en las prácticas cotidianas y servir como un sistema de significado para la sociedad; ${ }^{5}$ es en este sentido que mi artículo ofrecerá una perspectiva pertinente para entender la manera en la que los chinos se han esforzado por utilizarlo para buscar significado y solidaridad en la era posconfucianista.

\section{La campaña para recitar a los clásicos confucianistas}

En los últimos años, la campaña para recitar a los clásicos confucianistas ha alcanzado la conciencia pública y se ha visto envuelta en controversias recurrentes. La campaña fue iniciada formalmente en China continental en 1993 por Wang Caigui,

\footnotetext{
${ }^{5} \mathrm{Yu}$, Xiandai Ruxue Lun, op. cit., p. 5.
} 
discípulo del ya fallecido intelectual neoconfucianista Mou Zongsan. Antes de la campaña continental, desde la década de 1980, Wang había estado promoviendo la recitación de los clásicos confucianistas y de otros clásicos, y en Taiwan millones de niños en edad escolar habían participado en la iniciativa. En un corto periodo, Wang había atraído a un buen número de seguidores en Beijing, Shanghái, Guangzhou y otras ciudades costeras. En 1995, nueve miembros del Comité Nacional de la Conferencia Consultiva Política del Pueblo Chino (zhongguo renmin zhengzhi xieshang buiyi quanguo weiyuanbui 中国人 民政治协商会议 全国 委员会), incluidos Zhao Puchu, Bing Xin y otras conocidas personalidades del ámbito de la cultura, presentaron una propuesta conjunta para la octava reunión nacional de la Conferencia, denominada "Un llamado urgente para establecer una corriente para la difusión de los clásicos entre los niños pequeños" (jianli younian gudian xuexiao de jinji buyu 建立幼年古典学校的紧急呼吁). La propuesta atrajo la atención del gobierno central y fue ejecutada por la Fundación China para el Desarrollo de la Juventud (zhongguo qingshaonian fazhan jijinhui 中国 青少年 发展 基金会), a través del Comité de la Comunidad y la Cultura (shequ yu wenhua weiynanbui 社 区与文化委员会). Bajo los auspicios del comité, rápidamente se formó un grupo consultivo que editó un texto y lo envió a la imprenta, Un texto de lectura de poemas antiguos chinos y prosas (zhonghua gushiwen duben 中华古诗 文 读本); 12 volúmenes en total. Hasta fines de 2003, la campaña había involucrado a más de cuatro millones de niños en edad escolar y beneficiado a más de 25 millones de adultos. ${ }^{6}$

En 2004, otra versión del texto clásico para jóvenes, Un texto para recitar a los clásicos culturales chinos para la educación primaria (zhonghua wenhua Jingdian Jichu jiaoyu Songben 中 华文化经典 基础教育诵本) fue publicado por la Editorial de la Educación Superior (gaodeng jiaoyu chubanshe 高等教育 出版社), filial del Ministerio de Educación. El editor nominal del texto fue el Instituto Chino de Confucio (zhonghua Kongzi Xuebui 中华孔子学会), con la colaboración de la Academia de

\footnotetext{
${ }^{6}$ Sobu.com, "Zhonghua gushiwen jingdian songdu gongcheng shishi wunian huiji sanqianwan ren" [El proyecto de lectura clásica ya cumplió cinco años y ha beneficiado a 30 millones de personas], 17 de diciembre de 2003.
} 
Cultura China de la Universidad de Beijing (beijiing daxue zbongguo wenhua shuyuan 北京大学 中国 文化 书院), la Escuela de Educación de la Universidad Normal de Beijing (beijing shifan daxue jiaoyu Xueyuan 北京师范大学教育学院) y la Asociación Confucianista Internacional (guoji ruxuelianbebui 国际 儒学 联合会). En realidad, quien estaba inmerso en los ideales confucianistas y estuvo a cargo del proyecto y la selección de los textos clásicos fue Jiang Qing (蒋庆). En su prefacio al libro, Jiang escribe:

La selección de Un texto para recitar representa la quintaesencia de los clásicos de la cultura china, que resulta obligatorio leer. Lo que llamo "Clásicos culturales chinos" son los clásicos que siempre habían sido reconocidos en la historia de China y que representaban los principios y las enseñanzas de los sabios y venerables (confucianistas); a saber, los Seis clásicos, los Cuatro libros, y las obras representativas de los grandes confucianistas, así como las antologías influyentes sobre el confucianismo. Lo que llamo "quintaesencia" son los valores primordiales que mejor reflejan los principios y enseñanzas de los sabios y venerables consagrados en los clásicos; es decir, las ideas más importantes que mejor pueden reflejar las formas eternas, los axiomas eternos. Lo que llamo "lectura obligada" quiere decir que son los clásicos más fundamentales, cuyo contenido, en un sentido cultural, todos los chinos deben conocer; es decir, si alguien no conoce el contenido de estos clásicos, ni ella ni él pueden ser vistos como chinos en un sentido cultural.

El propósito directo de la edición de Un texto para recitar es proporcionar a todos los niños en China, el libro de texto más básico para recitar a los clásicos de la cultura china. El renacimiento de la cultura china tiene que empezar con los niños. A través de recitar a los clásicos de la cultura china se planta en los niños las semillas de los principios y las enseñanzas de los sabios y los venerables para que, cuando crezcan, puedan entender de manera natural las ideas sobre la autoeducación y la realización personal articuladas por los sabios y los venerables a lo largo de la historia; es decir, las enseñanzas sobre "la sabiduría interior y la majestad exterior" (neisheng waiwang 内圣外王), sobre la culminación personal y la de los demás (Chengji Chengrou 成已成物), sobre el conocimiento de la naturaleza humana y del cielo (Zhixing Zhitian 知性知 天); es más, los niños pueden integrar las sabias enseñanzas al proceso de crecimiento de su vida y participar activamente en la gran creación de la historia y la cultura y, al final, esforzarse por apreciar y fusionarse con la transformación del cielo y de la tierra. ${ }^{7}$ 
Notablemente, todas las piezas seleccionadas en el texto de Jiang Qing provienen de los clásicos confucianistas, mientras que los clásicos de otras tradiciones, como las de Laozi y Zhuangzi del taoísmo, Han Feizi del legalismo, etcétera, están excluidos. La explicación de Jiang es que las enseñanzas taoístas están saturadas con tácticas y esquemas políticos y, por lo tanto, ejercerían mala influencia en los niños. En una entrevista con The Bund (Waitan buabao 外滩画报), ${ }^{8}$ Jiang Qing sugirió que hoy en día el mayor problema que tiene China es el resquebrajamiento de la moralidad, y que haría todo lo posible para promover que los niños recitaran a los clásicos confucianistas debido a que la moral China existía en la tradición confucianista. El 8 de julio de 2004, poco después del lanzamiento del libro de texto de Jiang Qing, Xue Yong, entonces estudiante de doctorado en la Universidad de Yale, publicó un artículo en Southern Weekend (Nanfang zhoumo 南方周末) titulado “Un conservadurismo cultural en marcha hacia el oscurantismo", ${ }^{9}$ donde critica vehementemente a Jiang por promover la recitación de los clásicos confucianistas, ya que para él esto significa "forzar a los niños de entre tres y 12 años de edad a memorizar aproximadamente 150000 caracteres que realmente no entienden”. Por esta razón, cree que el esfuerzo de Jiang es "un movimiento para aturdir a la gente" bajo la bandera del "conservadurismo cultural”, y lo llamó "oscurantismo cultural”.

Días después, Qiu Feng, un investigador liberal independiente, publicó en el diario Southern Metropolis Daily (Nanfang dushi bao 南方都市报) el artículo “El oscurantismo disfrazado de modernización" 10 para contraatacar las críticas de Xue. Relató que, desde principios del siglo xx hasta la fecha,

ta de la editora). Jiang Qing, Zhonghua wenhua jingdian jichu jiaoyu songben [Un texto para recitar a los clásicos de la cultura china en la escuela primaria], Beijing, Gaodeng jiaoyu chubanshe, 2004.

${ }^{8}$ Waitan huabao [The Bund], 18 de enero de 2005.

${ }^{9}$ Xue Yong, "Zouxiang mengmei de wenhua baoshou zhuyi" [Un conservadurismo cultural en marcha hacia el oscurantismo], Nanfang zhoumo [Southern Weekend], 8 de julio de 2004.

${ }^{10}$ Qiu Feng, "Xiandaihua waiyi xia de mengmei zhuyi" [El oscurantismo disfrazado de modernización], Nanfang dushi bao [Southern Metropolis Daily], 13 de julio de 2004. 
[...] la iconoclasia mayorista ya se ha convertido en una ideología instintiva para el chino moderno. En aquellos años había personas a quienes no se les insistía en que leyeran libros chinos debido a que [pensaban] que lo que habían enseñado los libros chinos era todo ignorancia y oscurantismo. Cien años después, estas voces todavía resuenan con claridad en nuestros oídos.

Qiu Feng continuaba argumentando que quienes criticaban la campaña de Jiang Qing para recitar a los clásicos

[...] erróneamente creyeron que podían emitir un juicio definitivo sobre la tradición China y los clásicos chinos y que su juicio no podría ser cuestionado. Esto ha demostrado una mentalidad hegemónica del monismo y el racionalismo, y la arrogancia y el egocentrismo del racionalismo son exactamente las características típicas del oscurantismo.

El intercambio de fuego entre Xue Yong y Qiu Feng encendió inmediatamente la controversia en torno del hecho de recitar a los clásicos confucianistas, y pronto Southern Weekend se convirtió en el escenario central de la lucha. El 22 de julio publicó simultáneamente cuatro artículos: “¿Qué es el oscurantismo?" ("Shenme shi mengmei” 什么 是 蒙昧) de Xue Yong, "¿Por qué no podemos leer a los clásicos?" ("Weishenme buneng dujing” 为什么不能读经) de Qiu Feng, “Ideas oscurantistas acerca de la educación y la tradición" ("Mengmei de jiaoyu linian yu chuantong guan” 蒙昧的教育理念与传统观) de Liu Haibo, y "La recitación, los clásicos y el conservadurismo" (“Beisong Jingdian yu baoshou zhuyi” 背诵, 经典与 保 守主义) de Zhu Guohua. En poco tiempo, otros periódicos, y muchos foros y blogs en línea, se unieron al debate, que en general se centró en dos aspectos: la metodología pedagógica en la recitación de los clásicos y los valores implícitos en las obras clásicas. El campo de la oposición argumentó que forzar a los niños a recitar a los clásicos confucianistas, sin comprenderlos, era una forma de oscurantismo pedagógico y añadiría una carga adicional a su estricto plan de estudios, mientras que los partidarios sostenían que los niños de entre tres y 12 años de edad eran buenos para memorizar y que no era necesario que entendieran los textos de inmediato. En cuanto a los valores morales transmitidos por los clásicos, el primero consideraba la recitación de los clásicos como un adoctrinamiento que podría 
endurecer o incluso envenenar el alma de los jóvenes, mientras que los segundos creían que la inmersión de los niños en los clásicos confucianistas rectificaría el resquebrajamiento moral y otros problemas sociales, y que a través de la rehabilitación de la cultura tradicional china podría restaurarse y protegerse su "carácter chino", el cual había sido erradicado por el traumático proceso de modernización desde principios del siglo XX.

La importancia de la controversia acerca de la recitación de los clásicos ha sido reconocida o cuestionada por los estudiosos de diversas disciplinas. Wang $\mathrm{Yi}$, un joven profesor de la Universidad de Chengdu, afirmó: “es una oportunidad perfecta para que la principal corriente de intelectuales y el público en general contemplen la tradición, la respeten y simpaticen con ella; y, por lo tanto, puede ayudar a remediar los prejuicios centenarios acerca de que el sistema de valores nativo es enemigo de la libertad y la democracia”. ${ }^{11}$ Sin embargo, Wang Binbin, de la Universidad de Nanjing, expresó una opinión diferente: que la polémica sobre la recitación de los clásicos fue un episodio más sobre un tema recurrente de la historia moderna de China que tuvo que ver con la ruptura de las normas sociales y la desorientación de los valores morales, y que desde entonces refleja la confusión moral y la ansiedad colectiva en un momento de transformación social radical. ${ }^{12} \mathrm{El}$ mismo Jiang Qing también ha hecho un comentario sobre dicha polémica:

Con relación a la controversia sobre la recitación de los clásicos confucianistas creo que tiene un significado simbólico; es decir, después de cien años de alienación y ausencia, la cultura confucianista se ha convertido gradualmente en un discurso público. Hoy en día incluso las personas con diferentes ideologías e "ismos" tienen que enfrentar esta cuestión [...] porque la vitalidad de una cultura radica en el hecho de que se trata de un discurso público. Ahora el confucianismo es un discurso público. Esto prueba que la tradición confucianista ha recuperado su vitalidad y ha puesto fin a su alienación y marginación de los últimos cien años. ${ }^{13}$

${ }^{11}$ Wang Yi, "Dujing beihou de baoshou zhuyi he yuanjiaozhi" [El conservadurismo y el fundamentalismo detrás de la "recitación de los clásicos"], Shu Wu [Bookhouse], núm. 10, 2004.

${ }^{12}$ Wang Binbin, "Jiudiao chongtan shuo 'dujing" [Volver al tema recurrente: sobre la recitación de los clásicos], Hebei ribao [Hebei Daily], 10 de diciembre de 2004.

${ }^{13}$ Jiang Qing, "Dujing, rujiao yu zhongguo wenhua de fuxing-yuandao tongren 2004 Beijing Jiang Qing xiansheng fangtan lu” [Leyendo a los clásicos, el confucianismo 
Si bien, durante la controversia, la campaña de Jiang Qing fue criticada por algunos académicos, había un buen número de intelectuales liberales que, debido a su antagonismo tradicional frente a los conservadores, sorpresivamente expresaban su simpatía y apoyo a Jiang Qing. Qiu Feng, ${ }^{14}$ Liu Haibo y Wang Yi, tradicionalmente, habían estado asociados con el ala liberal que se supone estaba en desacuerdo con el conservadurismo; sin embargo, durante la polémica, todos acudieron en ayuda de Jiang Qing y sus aliados. Esto contrasta agudamente con el modelo convencional de todo el siglo xx, cuando los intelectuales de mentalidad occidental generalmente eran vistos como enarbolando las banderas de la "democracia" y de la "ciencia", debido a que el Movimiento del 4 de Mayo y cualquier cosa que fuera tradicional era etiquetada como "feudalismo" y "atraso", y pisoteada bajo sus pies.

\section{El renacimiento de las academias confucianistas}

Las academias confucianistas tradicionales se originaron a mediados de la dinastía Tang (618-907) y funcionaron principalmente como escuelas oficiales y bibliotecas donde los eruditos podían enseñar y estudiar los clásicos confucianistas y los libros eran recopilados y conservados. La dinastía Song del Norte (960-1127) atestiguó la época dorada de las academias confucianistas, ya que durante dicha dinastía se establecieron muchas academias con el apoyo del gobierno. A diferencia de la dinastía Tang, durante la dinastía Song las academias eran por lo general entidades privadas, construidas lejos de las ciudades o pueblos, cada una tenía su propia estructura administrativa y de enseñanza y era económicamente independiente; por lo tanto, los estudiosos confucianistas fueron provistos de un ambiente tranquilo en el que sus miembros podían participar en los estudios y la contemplación sin las restricciones y las distraccio-

y el renacimiento de la cultura china. Una entrevista con Jiang Qing realizada por colegas de Yuan Dao en Beijing en 2004], 2004.

${ }^{14}$ Durante los últimos años, Qiu Feng ha cambiado de manera decisiva su perspectiva intelectual del liberalismo al tradicionalismo, para posicionarse firmemente como el principal orador favorable a la restauración del confucianismo. 
nes mundanas. Aunque durante la dinastía Song del Sur (11271279) las academias confucianistas continuaron creciendo (11301200), la restauración de la Academia de la Gruta del Venado Blanco de Zhu Xi (bailudong shuyuan 白鹿洞书院) fue el suceso más significativo. Este sistema de academias, en su calidad de establecimientos privados, se debilitó bajo la dinastía Yuan (12711368) y todas ellas fueron puestas bajo el control gubernamental para convertirlas en escuelas preparatorias para los exámenes imperiales. Las dinastías Ming (1368-1644) y Qing (1644-1911) revivieron el sistema shuyuan, y el número de academias durante la dinastía Qing incluso sobrepasó al de todas las dinastías anteriores; sin embargo, la mayoría no asumió las mismas funciones y características que las academias Song. En medio de las crisis ocasionadas por las intromisiones de las potencias occidentales, el emperador Guangxu (光绪), de la dinastía Qing, emitió un decreto en 1901 para abolir formalmente el sistema de academias y la mayoría de ellas fueron convertidas en escuelas modernas al estilo occidental. ${ }^{15}$ Así, el milenario sistema de academias tradicionales confucianistas llegó a su fin.

Durante el periodo de la República (1911-1949), Xiong Shili, Liang Shumin y Ma Yifu - la llamada primera generación de neoconfucianistas - estaban fuertemente comprometidos con la preservación de la esencia de la tradición confucianista, y una de sus medidas fue el establecimiento de academias que se apegaran al modelo de la academia tradicional confucianista. Durante el periodo de la guerra sino-japonesa (1937-1945), Liang Shumin y Ma Yifu crearon la mianren shuyuan (勉仁书院 Academia Mianren) y la fuxing shuyuan (复性书院 Academia Fuxing), respectivamente, en la provincia de Sichuan, para educar a los estudiantes jóvenes a través de los clásicos confucianistas. Después de un corto periodo, ambas academias fueron cerradas debido a una serie de razones y a la situación sociopolítica que imperaba. A principios de la década de 1950, Qian Mu, supuestamente otro neoconfucianista de la primera generación, estableció una academia confucianista en Hong Kong, la xinya sbuyuan (新亚书院 La Nueva Academia Asia); sin embargo, de-

${ }^{15}$ Deng Hongbo, Zhongguo shuyuan Shi [Historia de las academias confucianistas en China], Shanghái, Dongfang chuban zhongxin, 2004, p. 330. 
bido al conflicto entre sus principios y los de la junta directiva de la escuela que posteriormente fue ampliada, Qian Mu renunció a su puesto en la academia, que con el tiempo se convirtió en una parte de la actual Universidad China en Hong Kong.

Antes de la República, las academias confucianistas tradicionales ejercieron tres funciones importantes: dar conferencias, presentar sacrificios y ofrendas, y preservar y, en algunos casos, imprimir libros. ${ }^{16}$ Además, estas academias por lo general tenían propiedades como bienes raíces o granjas, cuya explotación les servía para generar ingresos para sus gastos diarios. La Academia Mianren, la Academia Fuxing y la Academia Xinya eran como las academias confucianistas tradicionales en el sentido de que todas fueron fundadas por grandes eruditos confucianistas bajo la aguda conciencia de restaurar la tradición confucianista en tiempos muy alienantes, pero también eran muy diferentes en el sentido de que no mantenían los ritos confucianistas tradicionales ni hacían esfuerzos para rescatar y conservar los libros confucianistas. Tampoco tenían propiedades para generar ingresos y ser autosuficientes; por ello, todas acabaron por ser patrocinadas por el gobierno y por otras instituciones, $\mathrm{y}$ a menudo se vieron obligadas a hacer concesiones relacionadas con sus principios, lo cual a la larga tuvo como resultado su propio fin.

En octubre de 1984, los ya desaparecidos filósofos Feng Youlan y Zhang Dainian, de la Universidad de Beijing, se coordinaron con numerosos académicos de otras universidades e instituciones para crear conjuntamente la Academia de la Cultura China (zhongguo wenhua shuyuan 中国 文化 书院), en Beijing, y formaron una entidad multiinstitucional dedicada a la educación y a la investigación académica. La academia tiene un doble propósito: rescatar y desarrollar los aspectos positivos de la herencia cultural mediante la enseñanza de la cultura tradicional china, y mejorar su investigación con el fin de facilitar su modernización a través de la introducción de las culturas extranjeras, la investigación de ellas y la realización de actividades de intercambio académico y de comunicación internacional.

${ }^{16}$ Liaowang Dongfang Zhoukai [Oriental Outlook], "Shuyuan xingqi biaozhi wenhua fuxing” [La restauración de las academias indica un renacimiento cultural], núm. 27, 2009. 
El cuerpo docente de la academia está conformado por profesores de filosofía y otras disciplinas relacionadas con las humanidades, y la academia se dedica a cultivar a los jóvenes investigadores nacionales y extranjeros, comprometidos con la investigación sobre la cultura, la filosofía, la historia y la literatura tradicional chinas. ${ }^{17}$ Sin embargo, esta Academia de la Cultura China difiere significativamente de las academias confucianistas tradicionales, ya que no es una institución "privada", no cuenta con instalaciones físicas, y de alguna manera funciona como una especie de instituto virtual de posgrado bajo los auspicios de la Universidad de Beijing; y más importante aún, en lugar de promover las enseñanzas confucianistas, está comprometida con la investigación académica de las humanidades en general.

El renacimiento de la shuyuan (书院 la academia) en los últimos años es más bien un fenómeno de carácter popular que ha ido arraigando discreta pero constantemente en la conciencia pública. Dependiendo de sus orígenes y funciones, estas academias, surgidas recientemente, pueden dividirse en tres grupos. El primero es el grupo shuyuan, creado por escritores contemporáneos. Destaca el hecho de que algunos de estos escritores son presidentes de sus respectivas asociaciones de escritores provinciales. Ejemplos de ello son wansongpu shuyuan (万 松浦 书院), creada por Zhang Wei, presidente de la Asociación de Escritores de la provincia de Shandong; bailu shuyuan (白鹿书 院), por Chen Zhongshi, presidente de la Asociación de Escritores de la provincia de Shannxi, y beiyang shuyuan (北洋书 院), por Feng Jicai, presidente de la Asociación de Escritores de la ciudad de Tianjin. ${ }^{18}$ Estas academias por lo general cuentan con establecimientos físicos, tienen la capacidad de movilizar recursos sociales, y a menudo se asocian con una determinada universidad o colegio con el fin de funcionar como un foro literario o una escuela de formación literaria. Casi no tienen ningún interés en los clásicos o en las enseñanzas confucianistas $\mathrm{y}$, como resultado, tienen muy poco en común con las academias confucianistas tradicionales.

${ }^{17}$ Baidu baike, "Zhongguo wenhua shuyuan" [La academia cultural de China].

${ }^{18}$ Liaowang Dongfang Zhoukai, op. cit. 
El segundo grupo de shuyuan nuevas se refiere a aquellas tradicionales que alguna vez fueron reducidas a reliquias históricas o centros turísticos, pero que ahora han sido revitalizadas o están en proceso de revitalización. Ejemplos de ello son yuelu shuyuan (岳麓 书院), songyang shuyuan (嵩阳 书院) y bailudong shuyuan (白鹿洞书院 la Academia de la Gruta del Venado Blanco), tres de las llamadas cuatro grandes academias. ${ }^{19}$ Durante la dinastía Qing, el número de academias tradicionales confucianistas alcanzó más de 7000, y el número de estructuras físicas de las academias tradicionales, incluso hoy en día, permanece en los cientos. La mayoría de ellas se han convertido en reliquias históricas, sitios turísticos, estructuras anexas a las escuelas, o edificios con cualquier tipo de propósito. Con el auspicio de la Universidad de Hunan, yuelu shuyuan funciona como una universidad de posgrado y es la única academia tradicional que conserva sus funciones pedagógicas. Incluso un nuevo museo de la shuyuan está siendo construido en sus instalaciones. A pesar de ello, ha renunciado a sus funciones tradicionales de hacer ofrendas y sacrificios a los sabios y de preservar los libros confucianistas. ${ }^{20}$

Mientras tanto, la Universidad de Zhengzhou, en la provincia de Henan, ha tomado notas de yuelu shuyuan y trata de utilizar los recursos de songyang shuyuan, situada cerca de Zhengzhou, provincia de Henan, para sus fines pedagógicos. Por otro lado, la escuela normal Jiujiang, en la provincia de Jiangxi, también ha patrocinado algunas de sus actividades estudiantiles en bailudong shuyuan, ubicada a 40 kilómetros de distancia; obviamente, en un riguroso intento por revitalizar algunas funciones de la shuyuan. ${ }^{21}$ Está claro que, incluso si las academias mencionadas pueden ser restauradas físicamente, es muy probable que sólo sea para que funcionen como centros secundarios de alguna universidad bajo un determinado sistema educativo moderno, muy lejos de su glorioso pasado confucianista.

A diferencia de las academias de "tipo escritor" y de "tipo universitario" mencionadas, las academias del tercer grupo traHenan.

${ }^{19} \mathrm{La}$ cuarta se llama yingtian shuyuan y se encuentra en Shangqiu, provincia de

${ }^{20}$ Liaowang Dongfang Zhoukai, op. cit.

${ }^{21}$ Idem. 
tan de conformarse a sí mismas a partir del modelo de las academias confucianistas Song-Ming y están firmemente comprometidas con una ambición ético-religiosa: diacrónicamente, para continuar con el daotong (道 统, el hilo-la transmisión del Tao) de la mano de los neoconfucianistas de Song-Ming, y sincrónicamente, para protegerse de las "herejías" y salvaguardar la integridad y la dignidad de la cultura china, siendo el cristianismo el candidato más accesible para ejercer dicha protección. ${ }^{22}$ Mientras que las academias tradicionales confucianistas a menudo estaban atrapadas entre lo "privado" y lo "oficial" (especialmente durante las dinastías Yuan y Qing), estas academias confucianistas de reciente creación son muy conscientes de su papel extraoficial y extragubernamental, y hasta ahora se han mantenido de manera efectiva fuera del radar del sistema oficial. En este sentido, Jiang Qing, sin duda el representante más importante del confucianismo contemporáneo en China continental, ha pasado al primer plano; su yangming jingshe (阳明 精舍, literalmente la Morada Yangming) puede considerarse un ejemplo perfecto de este tipo de academia.

Construida en 1996, la Morada Yangming se encuentra en un pequeño pico, aproximadamente a dos horas de Guiyang, la capital de la provincia de Guizhou, y se integra armoniosamente con el entorno. Su ubicación remota proporciona un ambiente ideal para el cultivo personal y la discusión académica, como fue el caso de las academias confucianistas tradicionales. La academia cuenta con instalaciones sencillas; en cada uno de sus complejos residenciales para huéspedes detenta el nombre de un ideal confucianista diferente. La planta superior del edificio principal está reservada para el debate, y en la planta baja se ha erigido una estatua de Confucio y unas tabletas grabadas con los nombres de sus seguidores más influyentes. La Morada Yangming cuenta con financiamiento privado y su misión es fundamentalmente de naturaleza académica. Jiang Qing, des-

${ }^{22}$ Esta controversia sobre la "herejía" fue destacada por el incidente de Qufu justo antes de la Navidad de 2010, cuando la intención de los grupos cristianos en Qufu para construir una iglesia de resplandeciente estilo gótico, no lejos del templo de Confucio, fue saboteada por las protestas del campo confucianista unido. Véase el informe de Associated Press, "Church Plan in Hometown of Confucius draws Protest”, Fox Neres, 25 de diciembre de 2010. 
pués de haberse retirado de un puesto de profesor en el Colegio de Administración de Shenzhen, pasa la mayor parte de su tiempo de verano en la Morada Yangming, donde puede leer libros, encabezar discusiones y recibir a discípulos y peregrinos. Hasta ahora, el gobierno lo ha dejado en paz..$^{23}$

La Morada Yangming está destinada a funcionar en su totalidad de conformidad con el espíritu de las academias tradicionales confucianistas, incluidas su organización, administración y operación. Jiang Qing quiere que su academia sea un lugar para el debate y el cultivo confucianista avanzado; en sus propias palabras, es un lugar para "leer, mantener el Tao y debatir" (dushu shoudao lunxue 读书, 守道, 论学) y no está abierto al público en general. Al igual que las academias confucianistas tradicionales, la Morada Yangming difiere significativamente de las escuelas oficiales, ya que no ofrece cursos de formación para desarrollar competencias profesionales ni diplomas a sus estudiantes; sin embargo, Jiang Qing está convencido de que a través de actividades académicas de alto nivel, la Morada Yangming es capaz de incidir en los estudiantes formados en el sistema oficial. Según él, esto debido a que la Morada Yangming ejerce la influencia espiritual del confucianismo y genera un significado simbólico para la cultura tradicional china; además, sostiene que lo que está haciendo "es salvaguardar el valor espiritual del confucianismo y reconstruir la hermenéutica confucianista". ${ }^{24}$

Con la Morada Yangming ganando cada vez más fama en los últimos años, han surgido otras academias confucianistas en todo el país; entre ellas, la Academia Pinghe (pinghe shuyuan 平和 书院), en Zhuhai; la Academia Yunshen (yunshen shuyuan 云深书院), en Wuhan, y la Academia Qufu (qufu shuynan 曲阜 书院), en Qufu. Todas han adquirido cierto grado de reconocimiento nacional y comienzan a ser destino para los aficionados confucianistas. El propósito de la Academia Pinghe se expresa en su breve introducción:

${ }^{23}$ Para una descripción de la Morada Yangming, véase Daniel A. Bell, “A Visit to a Confucian Academy”, Dissent, 22 de septiembre de 2008.

${ }^{24}$ Jiang Qing, "Jiang Qing Xiansheng Tan shuyuan Jianshe ji Ruxue Chuancheng” [Jiang Qing en la Academia de Desarrollo y Transmisión del Conocimiento del Confucianismo]. 
En la antigua China, shuyuan (la academia) fue el lugar donde se transferían la enseñanza confucianista y el Tao. Su objetivo era cultivar la virtud de una persona y transmitir el aprendizaje confucianista. El nombre [de shuyuan] se originó en la dinastía Tang, y fue establecido plenamente durante la dinastía Song, pero disminuyó junto con el declive de la cultura china en los tiempos modernos. Para construir una sociedad armoniosa es imprescindible revitalizar la cultura china, y para revitalizar la cultura china, shuyuan debe ser el camino. La Academia Pinghe fue creada con este propósito particular. ${ }^{25}$

Como se observa en su introducción general, la Academia Yunshen tiene un propósito similar. Su fundador, Sun Xingjian, escribe: "necesitamos miles, decenas de miles de shuyuan para transmitir la cultura china" y, por otra parte, "se espera que la Academia Yunshen, directa o indirectamente, pueda tener éxito, formar caballeros ejemplares imbuidos en el espíritu de la justicia, el amor, la sabiduría y la originalidad" ${ }^{26}$ De la misma manera, la Academia Qufu destaca tanto la transmisión de la cultura china como el cultivo personal.

A través de la enseñanza sistemática de los clásicos confucianistas y el humanismo tradicional [la Academia Qufu] tiene como función la formación de jóvenes investigadores que se especialicen en el aprendizaje de Estado (gиохие 国学), a fin de que puedan heredar y promover la cultura china y estar al servicio de la armonía social y el desarrollo de la cultura tradicional. ${ }^{27}$

Sin embargo, de acuerdo con Jiang Qiang, no es posible asumir que todas las academias confucianistas autoconcebidas siguen los lineamientos marcados por el espíritu de las academias confucianistas Song-Ming; para ello, afirma Jiang, una academia debe cumplir varias condiciones. En primer lugar, contrariamente al sistema de educación "oficial", tiene que ser de naturaleza "privada" o "popular". En segundo lugar, en lugar de enseñar conocimientos específicos, la principal función de la academia confucianista es transmitir el Tao (camino) ya

${ }^{25}$ La traducción del chino al inglés es de Yong Chen (nota de la editora). Pinghesy. com, "Pinghe shuyuan zongzhi”. Desafortunadamente, desde 2012 este sitio no está disponible para consulta. Yunshen].

${ }^{26}$ Sun Xingjian, "Yunshen shuyuan zongzhi" [Los principios de la Academia

${ }^{27}$ Véase “Qufu shuyuan zongzhi”, en qfrl.org. La Academia Qufu también se conoce con el nombre de Academia Nacional de Aprendizaje Qufu. 
que una academia es un lugar solemne y sagrado representado por el sentido último de la santidad; la colocación de la estatua confucianista y las tablas de sus discípulos en cada academia simboliza la transmisión del Tao confucianista. En tercer lugar, la academia confucianista no es sólo un lugar para el debate teórico, sino también un lugar para que los discípulos confucianistas busquen la iluminación (wudao 悟道) y la verificación mutua (xianghu yinzheng 相互. 印证), ya que cualquier tipo de actividad espiritual requiere de la verificación mutua de los colegas. ${ }^{28} \mathrm{El}$ ideal confucianista de Jiang Qing no sólo se limita a la reconstrucción de las academias confucianistas; para él, eso es sólo un paso hacia una revitalización holística de la civilización confucianista (rujiao 儒教). ${ }^{29}$

En los últimos cien años o más, el confucianismo ha sufrido enormes destrucciones. Para una cultura o una religión, la esencia primordial son sus enseñanzas y valores fundamentales, y cuando estas enseñanzas y valores se terminan [la cultura-religión] no puede ser revitalizada. Las formas externas, ya sean formas de organización o formas de educación, han de poner en práctica los principios y valores de la cultura china, y no adquieren importancia por sí mismos. Lo que más ha sido destruido del confucianismo en los últimos cien años es su sistema de valores. ${ }^{30}$

Con el fin de revitalizar la civilización confucianista (rujiao 儒教), Jiang Qing ha esbozado dos enfoques: el primero es un enfoque “ascendente" (shangxing luxian 上行 路线) para reconstruir la "política regia” (wangguan xue 王官 学), es decir, para confucianizar el sistema político, y el segundo es un enfoque “descendente" (xiaxing luxian 下行 路线) para reconstruir una sociedad confucianista de carácter popular; esto es, la reconstrucción de una sociedad de ritos y música (confucianistas) para la China moderna. ${ }^{31}$ Para Jiang Qing, la parte más importante, op. cit.

${ }^{28}$ Jiang, "Jiang Qing Xiansheng Tan Shuyuan Jianshe ji Ruxue Chuancheng”,

${ }^{29}$ Para Jiang Qing, rujiao va más allá del significado de "confucianismo como religión” y encarna la quintaesencia de la civilización china per se. Sin embargo, como él mismo lo afirma, por conveniencia teórica algunas veces utiliza rujiao como "la religión de Confucio".

${ }^{30}$ Jiang, "Jiang Qing Xiansheng Tan Shuyuan Jianshe ji Ruxue Chuancheng”, op. cit. La traducción del chino al inglés es de Yong Chen (nota de la editora).

${ }^{31}$ Idem. 
más que revitalizar la forma externa del confucianismo, es restaurar su sistema de valores y sus enseñanzas. En este sentido, la restauración de las academias confucianistas es sólo un paso en el acercamiento descendente de Jiang Qiang para revitalizar la civilización confucianista.

\section{La restauración de los ritos confucianistas}

Tradicionalmente, los ritos formaban una parte muy importante del sistema confucianista. El enfoque confucianista para promover rituales no se basaría en el castigo. Una cita famosa de las Analectas (2.3) ha dejado muy clara esta cuestión: "Dirige a la gente a través de reglamentos y mantenlos ordenados con castigos y evitarán los castigos, pero no tendrán un sentido de la vergüenza. Dirígelos con el poder moral y mantenlos en orden por medio de rituales y desarrollarán un sentido de la vergüenza y se corregirán a sí mismos". ${ }^{32}$

El resurgimiento de los ritos confucianistas tradicionales se ha manifestado en varios aspectos: el culto y el sacrificio a Confucio, la ceremonia matrimonial confucianista, y la ceremonia del "estreno de los pinceles". El culto a Confucio había sido durante mucho tiempo el culto estatal de la China tradicional y fue sólo hasta el siglo xx que la práctica fue interrumpida por el triunfo de la "democracia" y la "ciencia". "El comienzo del siglo XXI ha visto la constante rehabilitación de esta tradición en Qufu y el gobierno local también se une al coro, con la esperanza de impulsar la industria del turismo. La boda confucianista, de carácter experimental, hizo su debut hace apenas tres años en Shenzhen, una ciudad cercana a Hong Kong, con un empuje de manos de Jiang Qing y sus discípulos más cercanos; por el contrario, la ceremonia del "estreno de los pinceles” está bastante generalizada. Muchos niños en edad escolar y sus padres participan en la ceremonia, aunque en este ritual la frontera entre lo confucianista y lo extraconfucianista prácticamente se ha borrado.

${ }^{32}$ The Chinese Classics, vol. 1: The Confucian Analects, tr. James Legge, Taipéi, SMC Publishing Inc., 1991, p. 146. 


\section{El culto a Confucio}

El culto a Confucio es una gran ceremonia de adoración que tiene lugar en el templo que lleva su nombre. Tradicionalmente, la gran ceremonia era conocida como "la ceremonia del Estado" e incluía cuatro actividades principales: música, canciones, danzas y rituales, todas estrechamente vinculadas entre sí. A partir de 1949 y durante las primeras cuatro décadas del reinado comunista en China, el culto a Confucio fue abolido oficialmente como superstición y feudalismo, mientras que en Corea del Sur, Taiwan y algunas regiones en el sudeste de Asia, donde el confucianismo todavía ejercía una gran influencia, la ceremonia se llevaba a cabo anualmente. En 1986, en un intento por impulsar la industria turística local, la ceremonia fue parcialmente resucitada por el gobierno de Qufu, lugar de nacimiento de Confucio; sin embargo, no fue sino hasta 2004 cuando el culto a Confucio fue cubierto ampliamente por los medios de comunicación, y el público en general pudo presenciar por primera vez la realización de la ceremonia a través de la retransmisión, en directo en circuito cerrado de televisión. Más importante aún: los funcionarios del gobierno de Qufu participaron activamente en la ceremonia, lo cual podía ser interpretado como una intervención oportunista en la misión del Partido Comunista para construir una "sociedad armoniosa" (bexie shehui 和谐社会). Desde 2004, la gran ceremonia se realiza anualmente de manera ostentosa en el Templo de Confucio; mientras tanto, probablemente con las mismas motivaciones del gobierno local de Qufu, muchos templos de Confucio en todo el país han restaurado este ritual.

En cuanto al significado y la importancia de la ceremonia del culto a Confucio, Yang Yitang, subdirector de la Oficina del Festival de la Cultura Confucianista, ha hecho algunas observaciones difícilmente cuestionables:

En primer lugar, la ceremonia consiste en sostener la antorcha para la transmisión de la cultura china; en segundo lugar, representa la oportunidad de escuchar las enseñanzas del sabio; en tercer lugar, se trata de un viaje a través de la historia del pueblo chino; en cuarto lugar, es una oportunidad para reconstruir la vena del espíritu nacional; en quinto lugar, es una ocasión para mostrar al mundo la determinación que tiene 
China de tener "un ascenso pacífico"; en sexto lugar, es un esfuerzo por buscar la sabiduría para lograr la unificación pacífica de los dos lados del Estrecho de Taiwan; en séptimo lugar, es para ganar el corazón y el alma de las decenas de millones de chinos que se encuentran en ultramar; en octavo, es un lenguaje común para reforzar la integración del desarrollo del Asia oriental y, por último, es leer un canon sagrado para la coexistencia armónica de la humanidad..$^{33}$

Aunque muy optimistas, los comentarios de Yang sobre la ceremonia del culto a Confucio de alguna manera resumen sucintamente la capitalización que ha hecho el Partido Comunista del nombre de Confucio; es decir, para utilizar el imaginario confucianista como un medio conveniente para proyectar un poder blando, debido a que la obsoleta ideología comunista sobre el progreso social ya no tiene ninguna influencia en las mentes del siglo XxI. Entre los nueve beneficios de la ceremonia mencionados por Yang, sólo tres puntos pueden relacionarse con la visión universalista de las enseñanzas de Confucio: el ascenso pacífico, la integración y la coexistencia armónica. Los otros seis puntos tienen un carácter nacionalista y aprovechan la mentalidad general de la sociedad china en un tiempo de caos, ansiedad, oportunismo y orgullo; todo ello resultado de los cambios fundamentales que se han producido en todas las dimensiones sociales.

\section{La ceremonia matrimonial confucianista}

El 22 de mayo de 2010 se celebró en Shenzhen una ceremonia matrimonial confucianista tradicional; el patrocinador de la ceremonia fue el Salón de Confucio el Sabio (kongsheng tang 孔圣堂). La boda es importante porque fue la primera ceremonia matrimonial confucianista en más de cien años, desde el desmantelamiento del confucianismo como un sistema institucional a finales de la dinastía Qing. La ceremonia se llevó a cabo estrictamente de acuerdo con el procedimiento y la regulación prescrita por el Libro de los Ritos ( $L i J i$ 礼记), que consis-

${ }^{33}$ Radio Nacional de China, "Kongzi wenhuajie fuzeren jiedu 'jikong dadian de xiandai yiyi”" [La persona encargada del Festival Cultural de Confucio habla sobre la importancia de la "ceremonia dedicada a Confucio"], 5 de septiembre de 2008. 
tía en varios rituales; entre ellos, "la recepción de la novia", "la entrada al salón", "el homenaje a los suegros", "el intercambio de regalos" y "el atado del nudo". Las aproximadamente ochenta personas directamente implicadas en la ceremonia incluyeron al presidente de la ceremonia, el anfitrión, el supervisor, los músicos, el diácono, la compañía de teatro, los cargadores de la silla de mano, etcétera. Por primera vez la ceremonia empleó a una comparsa de oratoria y un coro confucianistas para ejecutar las odas del Libro de Poesía (Shi Jing 诗经) y algunos otros himnos solemnes tradicionales. La ceremonia ha sido ensalzada como una integración perfecta entre la tradición y la modernidad, en el sentido de que no sólo está conformada de acuerdo con el principio de los ritos confucianistas, sino que también atiende al gusto estético moderno. Algunos simpatizantes confucianista han dado incluso un paso más para aplaudir la ceremonia como el mejor ejemplo de la "transformación creativa" de la tradición confucianista y, más importante aún, afirman que la promoción y organización de la boda confucianista tradicional podría representar una gran contribución a la reconstrucción de la cultura familiar china, al mejoramiento de la tradición y la costumbre popular, y al establecimiento de una sociedad armoniosa. ${ }^{34}$

Es necesario señalar que el presidente de la ceremonia de la boda, Zhou Beichen, es el ejecutivo del Salón de Confucio el Sabio y, casualmente, uno de los discípulos de Jiang Qing. Autoproclamado como el espacio sagrado del confucianismo contemporáneo, se afirma que la misión principal del Salón de Confucio el Sabio es "evangelizar el camino sagrado, y exponer la enseñanza ortodoxa; cambiar las costumbres populares, $y$ restaurar y transformar la tradición; restablecer las creencias, y mejorar la moral" (chuanbo shengdao, jiangming zhengxue; yifeng yisu, fugu genghua; chongjian xinyang, zhenxing daode 传播圣道, 讲明正学; 移风易俗, 复古更化; 重建 信仰, 振兴道德). ${ }^{35}$ El Salón de Confucio el Sabio fue construido al

${ }^{34}$ Tencent.com, “'Kongshengtang rujia hunli’ Shenzhen zhengshi dengchang” [Una boda confuciana de Kong Sheng Tang se lleva a cabo en Shenzhen].

35 “Rujia daochang 'kongshengtang' yu 09 nian 8 yue zai Shenzhen zhengshi jiancheng” [Kong Sheng Tang - el lugar sagrado del confucianismo se ha establecido en Shenzhen, agosto de 2009]. 
estilo de una academia confucianista tradicional y consta de varias salas con los nombres de los ideales confucianistas. En el centro de la sala principal está la tabla "del cielo, la tierra, el emperador, los padres y el maestro” (tian dijun qin shi 天地君亲 师) y la estatua de Confucio. En su calidad de plaza sagrada del confucianismo, el Salón de Confucio el Sabio está destinado a servir como base para la difusión de las enseñanzas de Confucio y para propiciar la intercomunicación y congregación de sus seguidores. Curiosamente, la etiqueta con el nombre de la sala, kongsheng tang (孔圣堂), fue escrita por Gao Zhanxiang, el ex viceministro del Ministerio de Cultura, lo que sugiere una sutil convergencia entre la esfera oficial y la popular en la recuperación del pasado para el servicio del presente.

\section{La ceremonia del "estreno de los pinceles"}

La ceremonia del "estreno de los pinceles" era un ritual tradicional con el cual los niños eran iniciados en la edad escolar. Cuando los niños llegaban a la escuela, el primer día de clases, su maestro les enseñaba los principios morales más fundamentales y más sencillos, así como las directrices básicas para la lectura y la escritura. Posteriormente, se les conducía al frente de la estatua de Confucio, tradicionalmente ensalzado como el primer gran maestro de China, donde rendían homenaje al sabio. Sólo después del ritual podían ser considerados formalmente como niños de la escuela; la ceremonia era vista como el momento para “romper la ignorancia” (po meng 破 蒙). En la China tradicional, la ceremonia del estreno de los pinceles era muy importante para una persona que estaba decidida a unirse en el futuro a la clase de los literatos. Junto con la abolición del sistema tradicional de exámenes y la adopción de escuelas de estilo occidental, a finales de la dinastía Qing, la ceremonia del estreno de los pinceles también fue abolida.

Sin embargo, en los últimos años, esta antigua ceremonia ha resurgido discretamente y muchos templos confucianistas son ahora el lugar ideal para celebrarla. La primera ceremonia para el estreno de los pinceles conocida en la época contemporánea se llevó a cabo en la primavera de 2004, en el templo confucianista 
de Guangzhou. La ceremonia incluyó siete pasos: vestir la ropa y la gorra escolar (zheng yigua 正 衣冠), cruzar el puente de las nubes azules (pingbu qingyun 平步青云), lavarse la cara con una toalla escolar (zhuangyuanjin xilian 状元 巾 洗脸), abrir la sabiduría con un pincel de cinabrio (zhusha kaizhi 朱砂 开 智), tocar la campana de la sabiduría (qiao qi zhong 敲启智钟), practicar el primer trazo de caligrafía (qimeng miaohong 启蒙 描红), y pedir un deseo bajo un árbol de laurel de oro (jinguishu xia xuyuan 金桂 树下 许愿). ${ }^{36}$ Más de 20 niños entre cuatro y siete años que estaban a punto de entrar al primer grado participaron en la ceremonia, la cual fue bien recibida tanto por los padres como por el público en general. Los medios de comunicación también proporcionaron una cobertura completa del suceso. Mientras que algunas personas criticaron la ceremonia como superstición o como el resurgimiento del feudalismo, la mayoría de la gente la aplaudió como una manera de exponer a los niños a la excelencia y vitalidad de la cultura tradicional en una época saturada por la cultura popular occidental, como la música pop, Hollywood, los videojuegos, Internet, la comida rápida, etcétera. Independientemente de la controversia, es obvio que la popularidad de la ceremonia del estreno de los pinceles una vez más ha puesto de relieve la importancia de la educación en la conciencia pública, que siempre ha sido un valor fundamental en las enseñanzas de Confucio.

\section{Conclusión}

Hay muchas definiciones de tradición y una de las más conocidas proviene de Edward Shils, sociólogo que hizo una amplia investigación sobre el tema:

La tradición -aquello que se transmite de generación en generaciónincluye objetos materiales, creencias sobre todo tipo de cosas, imágenes de personas y actividades, prácticas e instituciones. Incluye edificios, monumentos, paisajes, esculturas, pinturas, libros, herramientas, máqui-

\footnotetext{
${ }^{36}$ Sina Neres, "Guangzhou zhongxuetong fugu xing kaibili, dai boshi mao bai kongfuzi” [Los niños de primaria reviven la ceremonia del estreno de los pinceles, se ponen la toga y veneran a Confucio en Guangzhou], 29 de febrero de 2004.
} 
nas; incluye todo lo que una sociedad posee en un momento determinado, que ya existía cuando sus actuales poseedores se encontraron con ello y que no es únicamente producto de un proceso físico en el mundo externo ni exclusivamente resultado de una necesidad ecológica y fisiológica. ${ }^{37}$

Una tradición puede estar viva o muerta, dependiendo del momento referencial sobre el que se hace el punto; por lo tanto, una tradición viva se refiere a una serie de creencias, objetos y costumbres que se originaron en el pasado, que se han realizado o creído a través del tiempo y que siguen teniendo relevancia durante el presente.

Llamar al confucianismo en la China del siglo XxI "una tradición viva", naturalmente hace resonar tonos discordantes entre las personas que de alguna manera han reconocido las vicisitudes del confucionismo en el turbulento siglo xx. La oposición más vehemente vendría de Joseph $\mathrm{R}$. Levenson, quien afirmó que "en algún lugar, a lo largo de los años, entre Matteo Ricci (1552-1610) y Liang Ch'i-Ch'ao (1873-1929), el confucianismo había perdido la iniciativa" y que "la visión del mundo [del confucianismo] había perdido su integridad y su relevancia contemporánea". 38 "Este proceso es lo que él llama la museización" 39 del confucianismo, una declaración muy conocida en el mundo de la sinología. La conclusión de Levenson podría ser cierta si no contáramos los últimos años del siglo XX y los primeros del siglo xxI, exactamente el periodo durante el cual el confucianismo ha estado tratando de salir del "museo" para volver a conectarse con la sociedad china.

De hecho, la revisión realizada en este artículo sugiere que los rastros de un renacimiento confucianista pueden identificarse dentro de las estratificaciones de la sociedad, algunos con matices oficiales, otros no. Con el fin de movilizar a la sociedad, el Partido Comunista se involucra en un giro pragmático de las doctrinas marxistas a los eslóganes que de cierta manera son nativistas, como el de “sociedad armoniosa” (hexie shehui 和谐 社会) y “ascenso pacífico” (beping jueqi 和平 崛起), y esto no

\footnotetext{
${ }^{37}$ Edward Shils, Tradition, Chicago, University of Chicago Press, 1981, p. 12.

${ }^{38}$ Levenson, Confucian China and its Modern Fate, op. cit., pp. IX, X.

${ }^{39} \mathrm{Idem}$.
} 
puede ocultar su inspiración confucianista, independientemente del contenido real. Más importante aún, esta relajación de la sujeción marxista sobre la sociedad ha creado, de hecho, un entorno favorable para la restauración de algunas costumbres y creencias tradicionales. El agresivo movimiento para establecer institutos confucianistas (kongzi xueyuan 孔子学院) en todo el mundo, la promoción a medias del culto a Confucio por los gobiernos locales, el movimiento de masas para que los escolares reciten a los clásicos confucianistas, la extrema popularidad de la poco ortodoxa interpretación de las Analectas por Yu Dan, el restablecimiento de la ceremonia del estreno de los pinceles, el renacimiento de las academias confucianistas, el reciente establecimiento de los festivales tradicionales como días festivos (el Festival Claro y Brillante o Festival Conmemorativo qingming jie 清明节, el Festival del Barco Dragón duanwu jie 端午节, el Festival de la Luna zhongqiu jie 中秋 节, por nombrar algunos), son testigos de que de una manera lenta pero constante, el confucianismo ha estado recuperando su territorio perdido.

Sin embargo, es necesario tomar nota de que las huellas del renacimiento confucianista, tan extravagantes como pueden ser, son esencialmente esporádicas, difusas y no están coordinadas a pesar de que en mayor o menor medida todas ellas pueden ser atribuidas a la rectificación tácita de la tradición de la ideología oficial. Este carácter esporádico, difuso y no coordinado está en agudo contraste con la misión inflexible de Jiang Qing, quien está decidido a encabezar una revitalización al por mayor del confucianismo. Los ideales de Jiang incluyen la confucianización del sistema político de China, que él cree que ha sido colonizado por la cultura occidental. Para Jiang Qing, las transiciones políticas deben basarse en los recursos culturales existentes a fin de lograr una legitimidad política de largo plazo. ${ }^{40}$

En lugar de ver el confucianismo como una tradición meramente cultural, religiosa y filosófica, como tradicionalmente lo

${ }^{40}$ Jiang Qing, Zhengzhi ruxue: dangdai ruxue de zhuanxiang, tezhi yu fazhan [Confucianismo político: transformación, características especiales y desarrollo del confucianismo contemporáneo], Beijing, Shanlian shudian, 2003, p. 39. 
hicieron los neoconfucianistas en el siglo xx, Jiang Qing procede a exponer con amplitud su significado político en un entorno hostil. El sistema político ideal para China, según Jiang, no es la democracia, sino más bien "la política regia" (wangdao zhengzhi 王道政治), debido a que está en conformidad con el espíritu del confucianismo. Jiang prescribe el contenido principal de "la política regia" como una legitimidad triple (wangdao de sanchong hefaxing 王道 的 三重 合法性): la legitimidad de las fuentes sagradas (chaoyue shensheng de befaxing 超越神圣的合法 性), la legitimidad de la continuidad histórica (lishi wenhua de befaxing 历史文化 的 合法性), y la legitimidad del respaldo de la gente al poder político y su adhesión a dicho poder (renxin minyi de befaxing 人心民意的合法性). ${ }^{41}$ De acuerdo con Jiang Qing, un sistema político es legítimo sólo si estos tres aspectos de la legitimidad están correctamente equilibrados.

Es sólo en este contexto que podemos comprender esencialmente el compromiso de Jiang Qing con una revitalización integral del confucianismo. No es de extrañar que él haya sido la mano que empujaba detrás de la campaña para recitar a los clásicos confucianistas, del renacimiento de las academias confucianistas, de la restauración de los ritos confucianistas, y de otras formas del movimiento confucianista renacentista. Todos estos movimientos caen en la categoría del enfoque "descendente” de Jiang Qing (xiaxing luxian 下行 路线) para la revitalización de la civilización confucianista (rujiao 儒教). ${ }^{42}$ Según él, este enfoque "descendente" se refiere a los esfuerzos por reconstruir una sociedad confucianista en cuanto a su aspecto cívico, que incluyen: la universalización de la educación de los clásicos confucianistas; la promoción de la moral social; el establecimiento de normas autónomas para la gente común; la difusión de las buenas costumbres; la prescripción de ritos diarios; el patrocinio del culto ancestral y el sacrificio; la construcción de academias para difundir las enseñanzas de Confucio; el man-

${ }^{41}$ Ibid., p. 39.

${ }^{42}$ Jiang Qing establece tres enfoques: el primero consiste en reconstruir el sistema exegético del confucianismo, el segundo en restablecer "la política regia" y, el tercero, en el enfoque "descendente", necesario para reconstruir una sociedad confucianista de carácter popular. Véase Jiang, "Dujing, rujiao yu zhongguo wenhua de fuxing-yuandao tongren 2004 Beijing Jiang Qing xiansheng fangtan lu”, op. cit. 
tenimiento de la estabilidad social, y la caridad social, como la creación de casas para ancianos, orfanatos, escuelas, bancos de alimentos, etcétera. ${ }^{43}$

Debido al clima político y al etos de la China contemporánea, el fuerte compromiso de Jiang Qing con el renacimiento holístico del confucianismo difícilmente puede escapar a la acusación de ser un "confucianismo fundamentalista" antioccidental; sin embargo, el hecho de que él haya surgido prácticamente como el "rostro" mismo de los movimientos para el resurgimiento confucianista sugiere que detrás de su supuesta postura fundamentalista algo necesita ser explicado. En otras palabras, ¿cómo entender estos movimientos de renacimiento confucianista con características fundamentalistas que han ido ganando cada vez más popularidad? ¿Y cómo entender la popularidad de Jiang Qing entre los seguidores de Confucio y su autoridad comúnmente reconocida en estos movimientos?

En este punto, el estudio antropológico del fundamentalismo puede ayudar a comprender el fenómeno del renacimiento confucianista en general y el fenómeno de Jiang Qing en particular. Anthony F. C. Wallace identifica las etapas por las que atraviesan todos los movimientos de revitalización:

1. El cambio social produce tensiones culturales entre los miembros de la sociedad.

2. La tensión cultural produce un intento de adaptación que conduce a la distorsión y al cambio en los patrones sociales y causa trastornos sociales.

3. Como respuesta a la tensión cultural, el fundamentalismo surge como una reafirmación ortodoxa de patrones culturales.

De acuerdo con este modelo, la abolición del confucianismo como sistema institucional a finales de la dinastía Qing puede considerarse la primera etapa de la "tensión cultural”; el fracaso del marxismo y el resquebrajamiento de la moralidad, la segunda etapa de "disrupción social”, y, por supuesto, el rena-

${ }^{43}$ Idem. 
cimiento confucianista, la tercera etapa. Los enfoques de Jiang Qing para revitalizar el confucianismo - a fin de reconstruir un sistema exegético para reinstalar "la política regia" y reconstruir una sociedad confucianista en cuanto a su aspecto cívico- de hecho se basan en su agudo diagnóstico de los problemas culturales, políticos y sociales de la China contemporánea, aunque sus recursos no sean necesariamente eficaces.

En los movimientos fundamentalistas, sus miembros se ven a sí mismos como los salvadores de la sociedad y están dispuestos a hacer grandes sacrificios por su causa. ${ }^{44}$ En consecuencia, el mundo es visto por ellos en términos de blanco y negro, y los comportamientos humanos como buenos o malos; además, para los fundamentalistas las ortodoxias son más que teoría: "Son cuerpos de creencias inviolables que todos los miembros deben suscribir para mantener sus posiciones dentro del movimiento". ${ }^{45}$ De hecho, el periplo personal de Jiang Qing es un vivo testimonio de este patrón fundamentalista. Anticipó su retiro como profesor en una universidad en Shenzhen, una próspera ciudad costera, y se retiró a una aldea remota y desolada en las montañas de la provincia de Guizhou, donde agotó todos los medios para recaudar fondos que apenas fueron suficientes para financiar la construcción de la Morada Yangming, y desde entonces ha tenido un estilo de vida puritano, todo en aras de actualizar el Tao. Los sacrificios que ha hecho sirven para justificar su posición fundamentalista y reforzar su autoridad moral. Irónicamente, el ideal neoconfucianista sobre la unidad entre el conocimiento y la acción (zbixing beyi 知 行合一) está perfectamente representado por la búsqueda fundamentalista de Jiang Qing.

Siempre hay dos caras en una misma moneda. La inquebrantable postura fundamentalista de Jiang Qing sugiere su alejamiento del mundo exterior, por una parte, y la solidaridad

${ }^{44}$ Ciertamente, la teoría de las tres etapas de Wallace es útil en el análisis de los recientes movimientos de resurgimiento confucianista en general, y de Jiang Qing en particular, a pesar de que después de él ha habido una gran cantidad de estudios sobre el fenómeno del fundamentalismo. Véase Anthony F. C. Wallace, "Cultural Composition of the Handsome Lake Religion”, en William N. Fenton y John Gulick (eds.), Symposium on Cherokee and Iroquois Culture (B. A. E. Bulletin), Washington, Smithsonian Institution, 1961, pp. 139-151.

${ }^{45} \mathrm{Idem}$. 
y la dinámica dentro de su grupo interno por la otra. No es casualidad que su Morada Yangming se haya convertido en el destino final de los peregrinos y aficionados confucianistas. Rodney Stark ha formulado una teoría interesante que ahora tiene una gran influencia en su estudio sociológico de la religión. "Los nuevos movimientos religiosos pueden tener éxito en la medida en que mantengan un nivel medio de tensión con relación a su entorno; y que sean estrictos, pero no demasiado"; y lo estricto en este sentido "se refiere al grado en el que un grupo religioso mantiene un estilo de vida separado y distintivo, o un alto estándar de moralidad en la vida personal y familiar, en áreas como la del vestido, la alimentación, la bebida, las diversiones, las costumbres de la época, la sexualidad, la crianza de los hijos y otras similares". ${ }^{46}$ La razón dada por Stark es bastante simple: la rigidez fortalece a los grupos religiosos; les permite identificar a los parásitos y con ello aumentar el nivel medio de compromiso en el grupo, que a su vez aumenta la credibilidad de la cultura religiosa. ${ }^{47}$

Está claro que la teoría de Stark se basa en la observación de los movimientos religiosos en las comunidades cristianas. Si no nos enredamos con las eternas polémicas sobre lo que cuenta como religión, su teoría, sin duda, nos ayuda a comprender a Jiang Qing y el renacimiento confucianista en general. Probablemente puede decirse que, precisamente a causa de la combinación de la filosofía fundamentalista de Jiang y su estricta autodisciplina moral, el ala conservadora de los movimientos para el restablecimiento del confucianismo está dotada con un toque de sinceridad religiosa; por ello, vemos a un grupo tras otro de jóvenes discípulos que dejan atrás sus comodidades y privilegios seculares, y cruzan ríos y montañas para llegar a un lugar remoto llamado Morada Yangming a solicitar orientación e inspiración de una persona a la que llaman maestro. Con Jiang Qing detrás de todo, los movimientos supuestamente esporádicos, difusos y descoordinados a favor del renacimiento confucianista, empoderados mediante un mecanismo de consolidación personalizado en una sola figura,

${ }^{46}$ Rodney Stark, "Why Religious Movements Succeed or Fail: A Revised General Model”, Journal of Contemporary Religion, vol. 11, núm. 2, 1996, pp. 133-146.

${ }^{47}$ Idem. 
se están convirtiendo no sólo una tradición viva, sino también en una religión viva.

Traducción del inglés: Carmen Arriola

Dirección institucional del autor:

Centro de Estudios de Asia y África

El Colegio de México

Camino al Ajusco 20

Pedregal de Santa Teresa

10740 México, D.F.

\ychen@colmex.mx

\section{Bibliografía}

Associated Press, "Church Plan in Hometown of Confucius draws Protest”, Fox Newes, 25 de diciembre de 2010. [foxnews.com/ world/2010/12/25/church-plan-hometown-confucius-drawsprotest/, consultado el 2 de julio de 2012.]

Baidu baike, “Zhongguo wenhua shuyuan” 中国文化书院 [La academia cultural de China]. [baike.baidu.com/view/309308.htm, consultado el 10 de agosto de 2013.]

Bell, Daniel A., "A Visit to a Confucian Academy", Dissent, 22 de septiembre de 2008. [dissentmagazine.org/online_articles/avisit-to-a-confucian-academy, consultado el 29 de junio de 2014.]

Deng Hongbo, Zhongguo shuyuan Shi 中国书院史 [Historia de las academias confucianistas en China], Shanghái, Dongfang chuban zhongxin, 2004.

Dirlik, Arif, "Guoxue/National Learning in the Age of Global Modernity”, China Perspectives, núm. 2011/1. [Chinaperspectives. revues.org/5371?file =1, consultado el 2 de julio de 2013.]

Jiang Qing, "Dujing, rujiao yu zhongguo wenhua de fuxing-yuandao tongren 2004 Beijing Jiang Qing xiansheng fangtan lu” 读经、儒 教与中国文化的复兴一原道 同人 2004 北京蒋庆先生访谈录 [Leyendo a los clásicos, el confucianismo y el renacimiento de la cultura china. Una entrevista con Jiang Qing realizada por colegas de Yuan Dao en Beijing en 2004], 2004. [confucius2000. $\mathrm{com} / \mathrm{admin} /$ list.asp? id =1408, consultado el 5 de junio de 2013.] Jiang Qing, "Jiang Qing Xiansheng Tan shuyuan Jianshe ji Ruxue Chuancheng” 蒋庆先生谈书院建设及儒学传承 [Jiang Qing 
en la Academia de Desarrollo y Transmisión del Conocimiento del Confucianismo]. [confucius2000.com/admin/list.asp?id $=2620$, consultado el 5 de agosto de 2012.]

Jiang Qing, Zhengzhi ruxue: dangdai ruxue de zhuanxiang, tezhi yu fazhan 政治儒学: 当代儒学的转向、特质与发展 [Confucianismo político: transformación, características especiales y desarrollo del confucianismo contemporáneo], Beijing, Shanlian shudian, 2003.

Jiang Qing, Zhonghua wenhua jingdian jichu jiaoyu songben 中华文 化经典基础教育诵本 [Un texto para recitar a los clásicos de la cultura china en la escuela primaria], Beijing, Gaodeng jiaoyu chubanshe, 2004.

Levenson, Joseph R., Confucian China and its Modern Fate: The Problem of Intellectual Continuity, Berkeley-Los Ángeles, University of California Press, 1968.

Liaowang Dongfang Zhoukai 瞭望东方周刊 [Oriental Outlook], “Shuyuan xingqi biaozhi wenhua fuxing” 书院兴起标志文化 复兴 (La restauración de las academias indica un renacimiento cultural), núm. 27, 2009.

Liu Haibo, “Mengmei de jiaoyu linian yu chuantong guan” 蒙昧 的 教育理念与传统观 [Ideas oscurantistas acerca de la educación y la tradición], Nanfang zhoumo 南方周末 [Southern Weekend], 22 de julio de 2004.

Pinghesy.com, "Pinghe shuyuan zongzhi". [pinghesy.com, consultado el 16 de septiembre de 2011.]

Qiu Feng, “Xiandaihua waiyi xia de mengmei zhuyi” 现代化外衣 下的蒙昧主义 [El oscurantismo disfrazado de modernización], Nanfang dushi bao 南方都市报 [Southern Metropolis Daily], 13 de julio de 2004.

Qiu Feng, “Weishenme buneng dujing”为什么不能读经 [نPor qué no podemos leer a los clásicos?], Nanfang zhoumo 南方周 末 [Southern Weekend], 22 de julio de 2004.

"Qufu shuyuan zongzhi". [qfrl.org, consultado el 18 de agosto de 2012.]

Radio Nacional de China, "Kongzi wenhuajie fuzeren jiedu 'jikong dadian de xiandai yiyi” 孔子文化节负责人解读 “祭孔大典的现 代意义” [La persona encargada del Festival Cultural de Confucio habla sobre la importancia de la "ceremonia dedicada a Confucio"], 5 de septiembre de 2008. [cnr.cn/2004news/whyl/200809/ t20080905 505092333.html, consultado el 27 de marzo de 2013.]

“Rujia daochañg 'kongshengtang' yu 09 nian 8 yue zai Shenzhen zhengshijiancheng” 儒家道场 “孔圣堂”于 09 年 8 月在深圳正 
式建成 [Kong Sheng Tang - el lugar sagrado del confucianismo se ha establecido en Shenzhen, agosto de 2009]. [kong.org.cn/ bbs2/MINI/default.asp?55-79101-0-0-0-0-0-a-.htm, consultado el 23 de octubre de 2012.]

Shils, Edward, Tradition, Chicago, University of Chicago Press, 1981. Sina Neres, "Guangzhou zhongxuetong fugu xing kaibili, dai boshi mao bai kongfuzi” 广州众学童复古行开笔礼 戴博士帽拜孔 夫子 [Los niños de primaria reviven la ceremonia del estreno de los pinceles, se ponen la toga y veneran a Confucio en Guangzhou], 29 de febrero de 2004. [news.sina.com.cn/s/200402-29/02442981837.shtml, consultado el 15 de abril de 2012.]

Sobu.com, "Zhonghua gushiwen jingdian songdu gongcheng shishi wunian huiji sanqianwan ren” 中华古诗文经典诵读工程实施五 年惠及三千万人 [El proyecto de lectura clásica ya cumplió cinco años y ha beneficiado a 30 millones de personas], 17 de diciembre de 2003. [learning.sohu.com/2003/12/17/47/article217054743. shtml, consultado el 12 de mayo de 2013.]

Stark, Rodney, "Why Religious Movements Succeed or Fail: A Revised General Model”, Journal of Contemporary Religion, vol. 11, núm. 2, 1996, pp. 133-146.

Sun Xingjian, "Yunshen shuyuan zongzhi" [Los principios de la Academia Yunshen]. [ys2557.com, consultado el 20 de mayo de 2013.]

Tencent.com, “Kongshengtang rujia hunli' Shenzhen zhengshi dengchang” “孔圣堂儒家婚礼” 深圳正式登场 [Una boda confuciana de Kong Sheng Tang se lleva a cabo en Shenzhen]. [lady.qq.com/a/20100603/000129.htm, consultado el 12 de julio de 2013.]

The Chinese Classics, vol. 1: The Confucian Analects, tr. James Legge, Taipéi, sMc Publishing Inc., 1991.

Waitan huabao 外滩画报 [The Bund], 18 de enero de 2005.

Wallace, Anthony F. C., "Cultural Composition of the Handsome Lake Religion", en William N. Fenton y John Gulick (eds.), Symposium on Cherokee and Iroquois Culture (B. A. E. Bulletin), Washington, Smithsonian Institution, 1961, pp. 139-151.

Wang Binbin, “Jiudiao chongtan shuo “dujing'”旧调重弹说 “读经” [Volver al tema recurrente: sobre la recitación de los clásicos], Hebei ribao 河北日报 [Hebei Daily], 10 de diciembre de 2004.

Wang Yi, "Dujing beihou de baoshou zhuyi he yuanjiaozhi" 读经 背后的保守主义和原教旨 [El conservadurismo y el fundamentalismo detrás de la “recitación de los clásicos”], Shu Wu 书屋 [Bookhouse], núm. 10, 2004. 
Xue Yong, “Zouxiang mengmei de wenhua baoshou zhuyi” 走向蒙 昧的文化保守主义 [Un conservadurismo cultural en marcha hacia el oscurantismo], Nanfang zhoumo 南方周末 [Southern Weekend], 8 de julio de 2004.

Xue Yong, “Shenme shi mengmei” 什么 是 蒙昧 [“¿Qué es el oscurantismo?”], Nanfang zhoumo 南方周末 [Southern Weekend], 22 de julio de 2004.

Yu Yingshi, Xiandai Ruxue Lun 现代儒学论 [Sobre el aprendizaje confucianista moderno], Shanghái, Shanghai renmin chubanshe, 1998.

Zhu Guohua, "Beisong Jingdian yu baoshou zhuyi” 背诵, 经典与 保守主义 [La recitación, los clásicos y el conservadurismo], Nanfang zhoumo 南方周末 [Southern Weekend], 22 de julio de 2004. 
